\title{
Integrative analysis of single-cell expression data reveals distinct regulatory states in bidirectional promoters
}

\author{
Fatemeh Behjati Ardakani 1,2,3, Kathrin Kattler ${ }^{4}$, Karl Nordström4, Nina Gasparoni ${ }^{4}$, Gilles Gasparoni ${ }^{4}$, \\ Sarah Fuchs ${ }^{4}$, Anupam Sinha ${ }^{6}$, Matthias Barann ${ }^{6}$, Peter Ebert ${ }^{2,3}$, Jonas Fischer ${ }^{1,2,3}$, Barbara Hutter ${ }^{7}$, \\ Gideon Zipprich ${ }^{8}$, Charles D. Imbusch ${ }^{7}$, Bärbel Felder ${ }^{8}$, Jürgen Eils ${ }^{8}$, Benedikt Brors ${ }^{7}$, Thomas Lengauer ${ }^{2}$, \\ Thomas Manke ${ }^{5}$, Philip Rosenstiel ${ }^{6}$, Jörn Walter ${ }^{4}$ and Marcel H. Schulz $z^{1,2,9,10^{*}}$
}

\begin{abstract}
Background: Bidirectional promoters (BPs) are prevalent in eukaryotic genomes. However, it is poorly understood how the cell integrates different epigenomic information, such as transcription factor (TF) binding and chromatin marks, to drive gene expression at BPs. Single-cell sequencing technologies are revolutionizing the field of genome biology. Therefore, this study focuses on the integration of single-cell RNA-seq data with bulk ChIP-seq and other epigenetics data, for which single-cell technologies are not yet established, in the context of BPs.

Results: We performed integrative analyses of novel human single-cell RNA-seq (scRNA-seq) data with bulk ChIP-seq and other epigenetics data. scRNA-seq data revealed distinct transcription states of BPs that were previously not recognized. We find associations between these transcription states to distinct patterns in structural gene features, DNA accessibility, histone modification, DNA methylation and TF binding profiles.

Conclusions: Our results suggest that a complex interplay of all of these elements is required to achieve BP-specific transcriptional output in this specialized promoter configuration. Further, our study implies that novel statistical methods can be developed to deconvolute masked subpopulations of cells measured with different bulk epigenomic assays using scRNA-seq data.
\end{abstract}

Keywords: Bidirectional genes, Single-cell RNA-seq, Epigenetics

\section{Background}

Promoters are key structures for a coordinated regulation of gene expression. The increasing number of largescale high-resolution epigenomic and RNA-sequencing technologies are leading to a deeper understanding of genome-wide promoter configurations. Recent studies show that the number of bidirectional promoters (BPs) in the human genome is much larger than previously anticipated [1-3]. Sensitive assays, such as sequencing of

\footnotetext{
*Correspondence: marcel.schulz@em.uni-frankfurt.de

${ }^{1}$ Excellence Cluster for Multimodal Computing and Interaction, Saarland Informatics Campus, Saarland University, Campus E1 7, Saarbrücken 66123, Germany

Full list of author information is available at the end of the article
}

nascent RNAs (GRO-seq) or $5^{\prime}$-ends of capped nascent RNAs (GRO-cap and Start-seq), allow the detection of unstable nascent RNAs produced at promoters, and have revealed more widespread bidirectional transcriptional initiation than previously recognized [4-6]. However, the exact classification of bidirectional or unidirectional promoters in a sample of interest is challenging, as it depends heavily on the sensitivity of the sequencing assay to recognize unstable, nascent RNAs $[7,8]$.

Recent studies discuss two types of bidirectional promoters. The first type concerns transcription of two RNAs in opposite direction from one core promoter, i.e., one promoter leads to bidirectional transcription [5, 9, 10]. In the second type, transcriptional initiation of both RNAs occurs at two distinct core promoters that are 
close to each other, but are oriented in reverse direction, thus sometimes termed divergent bidirectional promoters. In this work we focus on bidirectional promoters that have two distinct core promoter elements that drive divergent transcription of two nearby genes.

BPs harbor overrepresented TF binding sites such as GABPA, MYC, YY1, NRF-1, E2F1 and E2F4 [11]. For example, the introduction of GABPA binding sites into unidirectional promoters leads to bidirectional expression in $67 \%$ of the cases [12]. Further, the sequence elements at some BPs function as inseparable units [13]. Other TFs prevent bidirectional expression, for example, promoters that show elongation in only one direction show enrichment of CTCF binding sites $[4,14]$. However, more research is needed to investigate how TF binding determines directionality of initiation and elongation at BPs [9].

It was recently shown that the two transcription start sites (TSSs) at a BP define a nucleosome-free region (NFR) between them. The size of the NFR may be an important structural element in BP regulation, determining the availability of binding sites for different TFs at the promoter and thus influencing gene expression strength as well as responsiveness to external stimuli $[5,6]$. The current results point to a model, where an independent Pol2 complex assembles at each TSS and initiates transcription, such that accurate phasing of the +1 and -1 nucleosomes at these BPs allows epigenetic regulation through HMs [4-6]. Comparisons between BPs and unidirectional promoters suggest that HMs associated with active gene expression exhibit a bimodal distribution at BPs and that upstream proximal enhancer marks may regulate downstream gene transcription $[6,14]$.

In summary, previous studies rely on the comparison of unidirectional against bidirectional promoters to learn about BP regulation. In this study, we take a different approach, making use of recent advances in single-cell sequencing and study expression of genes at BPs in individual cells to learn about their regulation. Recent developments in single-cell genomics allow the measurement of RNA expression in individual cells with a similar accuracy as compared to bulk-sequencing of RNAs $[15,16]$. This advance has been used to define previously overlooked cell types and expression heterogeneity, e.g., [17].

We used novel and previously produced single-cell RNA-seq (scRNA-seq) data for HepG2 and K562 cells to investigate the expression behavior of genes at a BP. We found that four reproducible expression categories exist in BPs and that in the majority of the cases, one gene at a BP shows much higher expression than the other one. Using high-resolution histone modification datasets produced at IHEC standards [18] by the DEEP consortium or made available by ENCODE [19], we find novel associations of different structural and epigenetic features in these categories.

\section{Results}

To understand the regulation of the two genes at a bidirectional promoter, we propose a novel approach to exploit RNA-seq data at the single-cell level, in contrary to the existing studies that rely on bulk RNA-seq data. Bulk RNA-seq masks gene expression across individual cells, and thus may hide interesting expression patterns of bidirectional gene pairs (Fig. 1a).

Figure $1 \mathrm{~b}$ illustrates examples of single-cell expression patterns in HepG2 cells for selected BPs. It can be noted that, for instance, the magnitude of expression of the $A L G 2, E C E 2$ gene pair alternates across the cells, meaning that in some cells $A L G 2$ is higher expressed than ECE2 and vice versa. Similarly, $A A M P$ and $P N K D$ genes exhibit this alternation, but more frequently. These observations motivated us to inspect such diversities in a systematic manner by forming an expression matrix specific to BPs for clustering analysis.

\section{Four states of transcription with distinct bidirectional characteristics}

We form an individual matrix of all BPs representing the single-cell expression of the gene located on the Watson strand (Watson matrix). Similarly, we construct the same matrix for the gene on the Crick strand (Crick matrix) (Fig. 1c). To simplify the follow-up analyses, we swap a row of the Watson matrix with the corresponding Crick row, if the average single-cell expression of the former is lower than the latter. In this way, for a given BP, we always keep the higher expressed gene $(\mathrm{H})$ on the right side and the lower expressed one (L) on the left. Next, we form the final swapped BP matrix, where the rows represent the bidirectional genes $(N=1242)$ and the columns represent the cells (twice the number of single cells); the first half of the columns represent cells' expression of $\mathrm{L}$ genes and the second half represent the same for $\mathrm{H}$ genes. Since, the combined matrix contains the joint expression information for both genes of a BP in each row, we used hierarchical clustering to group the BPs according to their similarity in single-cell expression patterns. This led to four distinct transcription states in both cell lines (Fig. 2a HepG2, and Additional file 1: Fig. S2A K562) with the following characteristics: (1) Bidirectional Lowly Expressed $(B L E)$, where both genes of a BP are lowly expressed, (2) Bidirectional Weak Difference (BWD), where the $\mathrm{H}$ gene is higher expressed than the $\mathrm{L}$ gene with a weak difference between the two, (3) Bidirectional Strong Difference $(B S D)$, where the $\mathrm{H}$ gene is much higher expressed than the $\mathrm{L}$ gene and higher than in $B W D$, (4) Bidirectional No 

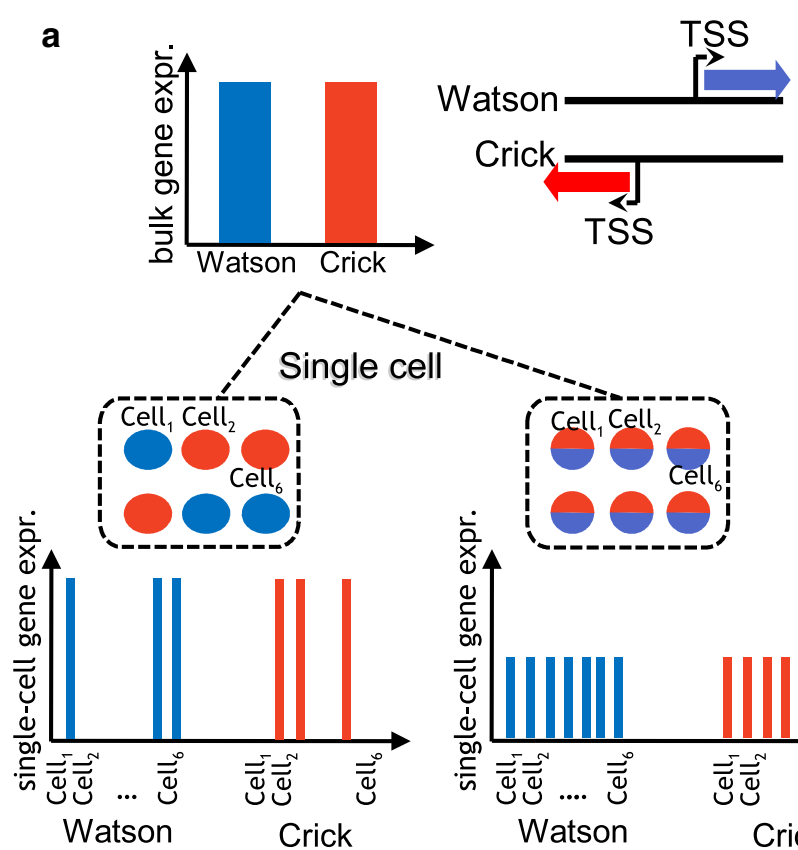

b
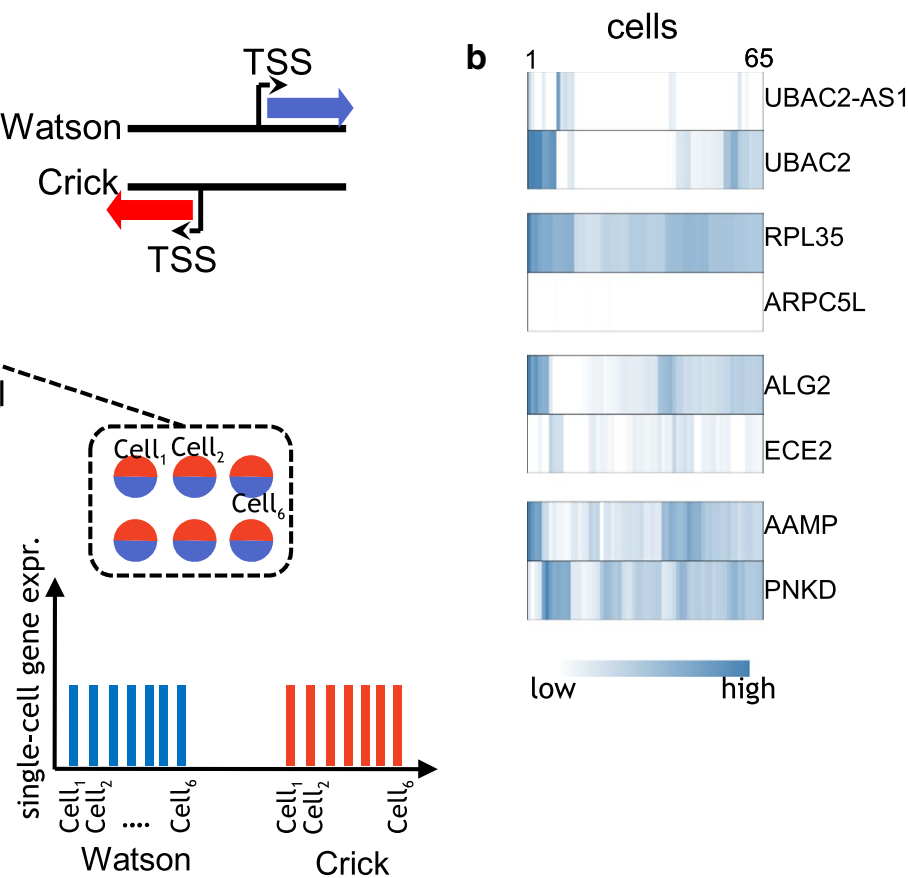

low high

C

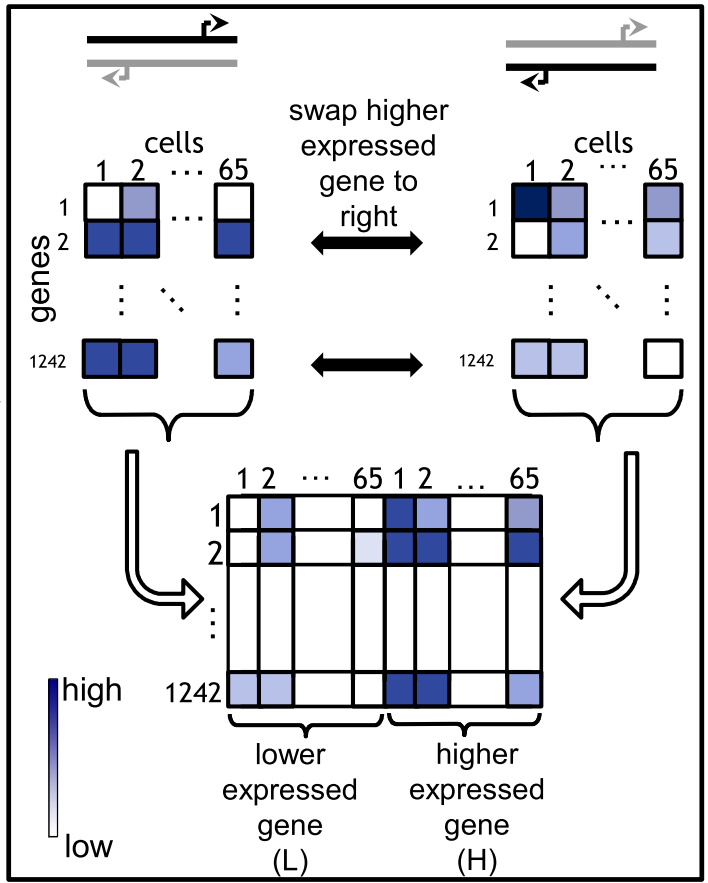

Fig. 1 Advantages of studying BPs at single-cell level. a An illustration of a BP, defined based on two genes located on opposing strands of DNA (Watson and Crick). Bulk RNA measurements at the BP may hide complexity of BP gene regulation. This is shown in the left single-cell expression scenario, where one of the genes is expressed and the other is silent in the same cell compared to the other scenario where single-cell expression agrees with bulk measurements. b Heatmaps of 65 single-cell RNA-seq expression measured in four bidirectional promoters (TPM, HepG2 cells). c After single-cell sequencing and estimating the gene expression of all genes in a cell, a set of 1242 BPs was extracted. Single-cell expression of either genes of a BP was arranged in two separate matrices for which the rows represent the BPs and columns the cells. Next, we swap the higher expressed gene to the matrix on the right and lower expressed one to the left. The resulting matrices are combined into one joint BP single-cell expression matrix 


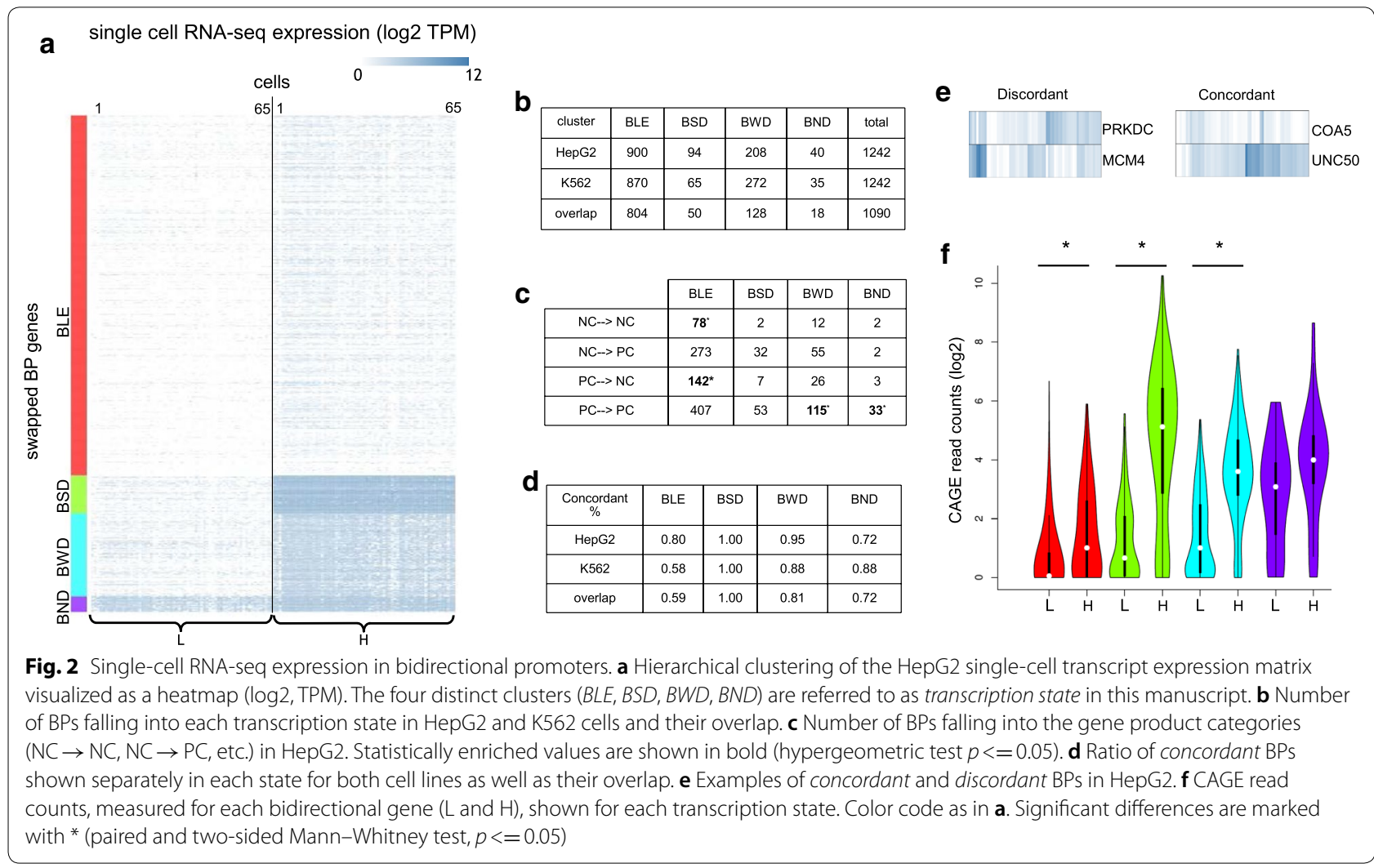

Difference $(B N D)$, where both genes of a BP are expressed relatively at the same rate.

The data regarding the frequency and type of BPs in each state are provided in Fig. 2 b, c. Figure $2 b$ reveals that most of the BPs associated to these states are common between the two cell lines (1090 out of 1242). We further investigated whether the transcription state was related to the type of genes encoded in a BP. We found that for both cell lines the $B W D$ and $B N D$ states are enriched with BPs (hypergeometric test, $p \leq 0.05$ ), where both bidirectional genes are annotated as protein-coding $(\mathrm{PC} \rightarrow \mathrm{PC}$, Fig. 2c, Additional file 1: Fig. S2B). On the other hand, the $B L E$ state is enriched with BPs of either two non-coding genes $(\mathrm{NC} \rightarrow \mathrm{NC})$ or where the $\mathrm{L}$ gene is annotated as protein-coding and the $\mathrm{H}$ gene as noncoding $(\mathrm{PC} \rightarrow \mathrm{NC})$.

The single-cell data allowed us to estimate the frequency of (concordant or discordant) gene signatures of BPs in all states for both cell lines (Fig. 2d, e). The BLE state was overall lowly expressed and due to stochasticity of expression, it is difficult to find a consistent pattern for this particular state. On the other hand, the BSD state consists of BPs where one gene's expression is always higher than the other, thus we obtained a concordant ratio of 1 . As expected, the $B N D$ state is showing some of the smallest concordant ratios, i.e., highest discordance, which points to the frequent alternations (switches) occurring in the expression of the genes in this state.

Figure $2 \mathrm{f}$ illustrates that the CAGE expression distributions follow the characteristics attributed to each cluster (similarly for the bulk RNA-seq and CAGE in K562 cell line, Additional file 1: Fig. S2C, D). However, it is worth mentioning that performing the clustering based on the bulk data, either RNA-seq or CAGE did not lead to a reproduction of the transcription clusters based on single-cell RNA-seq, due to measuring a population of cells in bulk assays (data not shown).

The representation used in Fig. 1d is concise, but it does not provide a suitable visualization to explore the associations between $\mathrm{L}$ and $\mathrm{H}$ genes in the same cell. Therefore, to quantitatively assess the relation between single-cell expression of bidirectional genes in these states, we computed, for each BP, the correlation between expression of $\mathrm{L}$ and $\mathrm{H}$ genes across single cells (Fig. 3a, data shown for both cell lines). The correlation analysis showed that the $B N D$ state has the highest correlation. On the contrary, the $B S D$ state revealed lower correlation, which suggests a more independent regulation of its bidirectional genes. To address which mechanism(s) are involved in driving such differences in regulation of BPs, we explored the following aspects: (1) structural features, (2) epigenetic signals, and (3) transcriptional regulatory elements. 


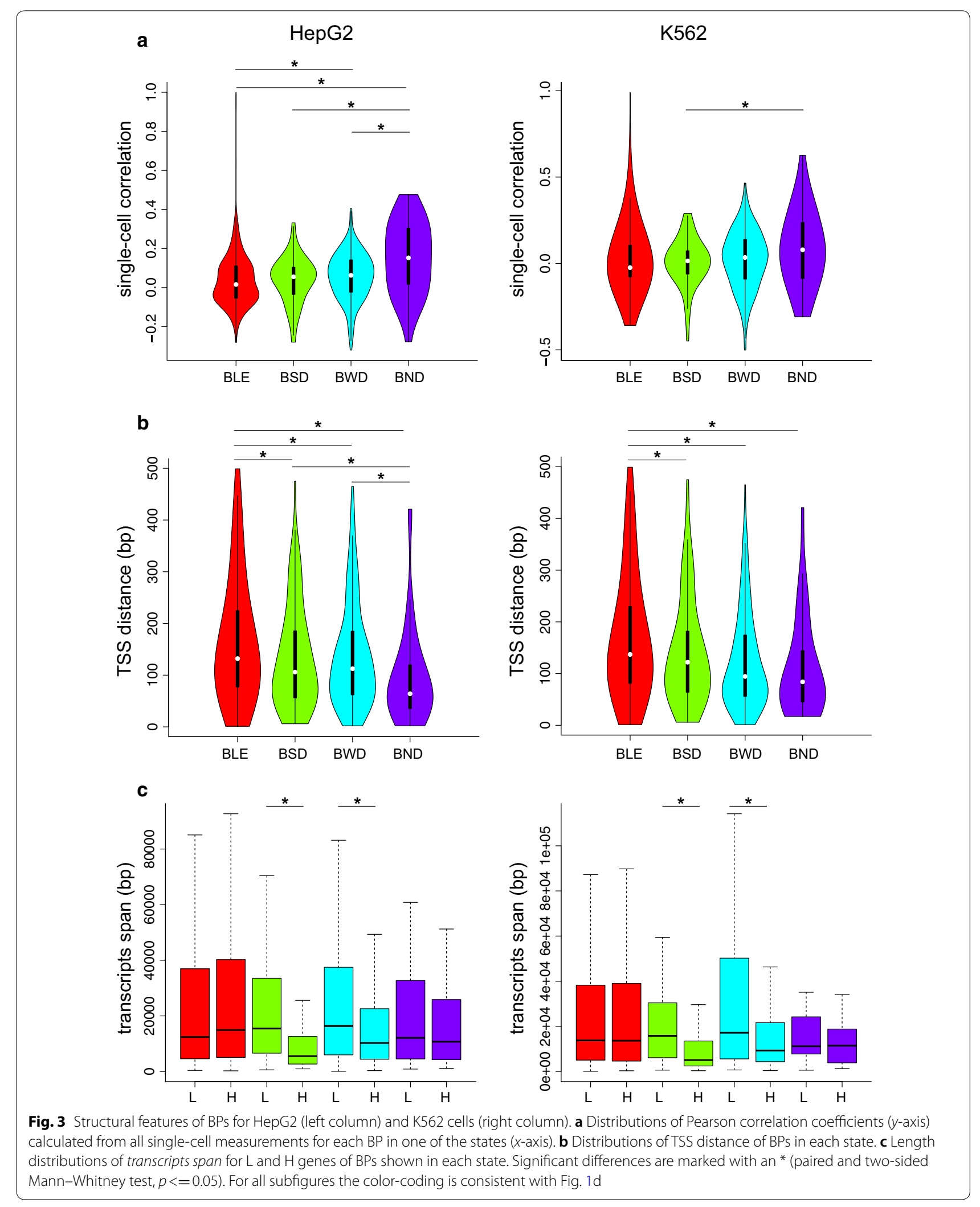




\section{Structural features associated with transcription states}

We first tested whether the distance between TSSs of bidirectional genes was associated with the transcription states. Figure $3 \mathrm{~b}$ depicts the distributions of TSS distances in each state for both cell lines. We observed that the $B L E$ state exhibits significantly larger TSS distances compared to the other states $(t$ test, $p \leq 0.001)$. On the contrary, the $B N D$ state had the smallest median distance (significant for HepG2, $t$ test $p \leq 0.05$ ). This observation together with the correlation analysis in Fig. 3a suggests that the smaller distance may influence recruitment of a common regulatory complex that facilitates the simultaneous regulation of both genes.

As the scRNA-seq protocol measures steady-state fully elongated mRNAs, we wondered whether the length of the transcribed region differs in the genes associated to the BPs. For this, we examined the region spanned by all transcripts originating from transcription start sites within $2 \mathrm{~kb}$ from the most $5^{\prime}$ TSS of a BP gene, a region we refer to as transcripts span (see "Methods"). Surprisingly, this length was significantly smaller (Mann-Whitney test, $p$ value $\leq 0.05$ ) for the $\mathrm{H}$ genes of states $B S D$ and $B W D$ compared to their counterpart L genes. Connecting this observation to the actual transcription expression depicted in Fig. 1d for these two states suggest that the expressions of $\mathrm{L}$ and $\mathrm{H}$ genes are inversely related to their transcripts spans in BPs. To elucidate whether this association holds for all genes or only BPs, we measured the transcripts span for all 63678 annotated genes in the human genome. We found no association of transcripts span with gene expression for all genes (Additional file 1: Fig. S2F), indicating that such structural configuration might be specific to BPs. As the estimated TPM values are derived from the exonic regions only, we further examined the transcript length by measuring the exonic region of all transcripts initiating within the $2 \mathrm{~kb}$ from the most $5^{\prime}$ TSS of a BP gene (Additional file 1: Fig. $\mathrm{S} 2 \mathrm{H}, \mathrm{I})$. We found a slight increase in TPM values for the larger genes, regardless of considering all genes or only BPs (Additional file 1: Fig. S2F).

We also investigated whether the difference in GCcontent could be involved in driving variation on the observed expression patterns, but we found no apparent differences (Additional file 1: Fig. S2G).

\section{Histone modification and DNasel patterns reflect the characteristics observed in transcription states}

To explore the role of epigenetics in transcription states observed in Fig. 1d, we produced seven histone modifications (H3K4me1, H3K4me3, H3K36me3, H3K27me3, H3K9me3, H3K27ac, and H3K122ac) and DNaseIseq data for HepG2 cells within the DEEP consortium. Further we obtained data for DNaseI-seq and all modifications, except H3K122ac, for K562 cells from [19]. Figure 4 depicts the normalized read counts measured around the TSSs of bidirectional genes stratified according to the transcription states for all HepG2 datasets (similarly, for K562 in Additional file 1: Fig. S3A). Generally, we observed that the epigenetics data show specific patterns related to these states. For instance, it is notable that the $B L E$ state had the lowest abundance for HMs associated with active promoters (H3K4me1/3, H3K36me3, H3K27ac, and H3K122ac) and highest for H3K27me3 and H3K9me3 that are mostly associated with repressed promoters [20]. On the other hand, the $B N D$ state exhibited the very opposite behavior to $B L E$, reflecting their expression characteristics observed in Fig. 1d.

Another interesting observation is the agreement of the elongation mark profiles, H3K36me3, with the transcripts span distribution shown in Fig. 3c. In general, the larger the increase in the H3K36me3 mark, the shorter the transcripts span for the gene. For instance, the BSD state that has the shortest transcripts span exhibits the sharpest increase in its H3K36me3 profile downstream of the H gene's TSS. This fits to the previous observation that the H3k36me3 mark increases gradually and peaks at the end of genes [21] and we can observe that general trend for the transcripts span on our data as well (Additional file 1: Fig. S3B).

In a recent study by Wang et el. [22], it has been shown that mRNA stability can be estimated using HM data at promoters. Previous research on genome-wide measurements of RNA half-lives suggested that lncRNAs exhibit a wide range of stabilities similar to that of protein-coding transcripts [23]. Therefore, we used the approach by Wang et al. to estimate which genes appear to be stable and unstable, with the idea that this could also explain differences in the gene expression behavior we observe in the different states. In brief, this method uses HM signals at promoters, as features, and gene expression measurements, as response, to learn a linear model that predicts gene expression. Using outlier analysis, genes that show lower (higher) expression as predicted are marked as unstable (stable) (see "Methods" and Additional file 1: Fig. S4A). The results show that the putative stable genes are significantly (hypergeometric test, $p \leq 0.05$ ) enriched in all the states except $B L E$ (consistent across both HepG2 and K562 samples). On the other hand, the $B S D$ state was significantly enriched in the putative unstable category, with $\sim 21 \%$ and $30 \%$ of its genes being inferred as unstable in HepG2 and K562 samples, respectively (Additional file 1: Fig. S4B).

The DNaseI-seq profile of the $B N D$ state revealed not only the highest signal, but also the widest spread around the TSS compared to the other states. This agrees with 


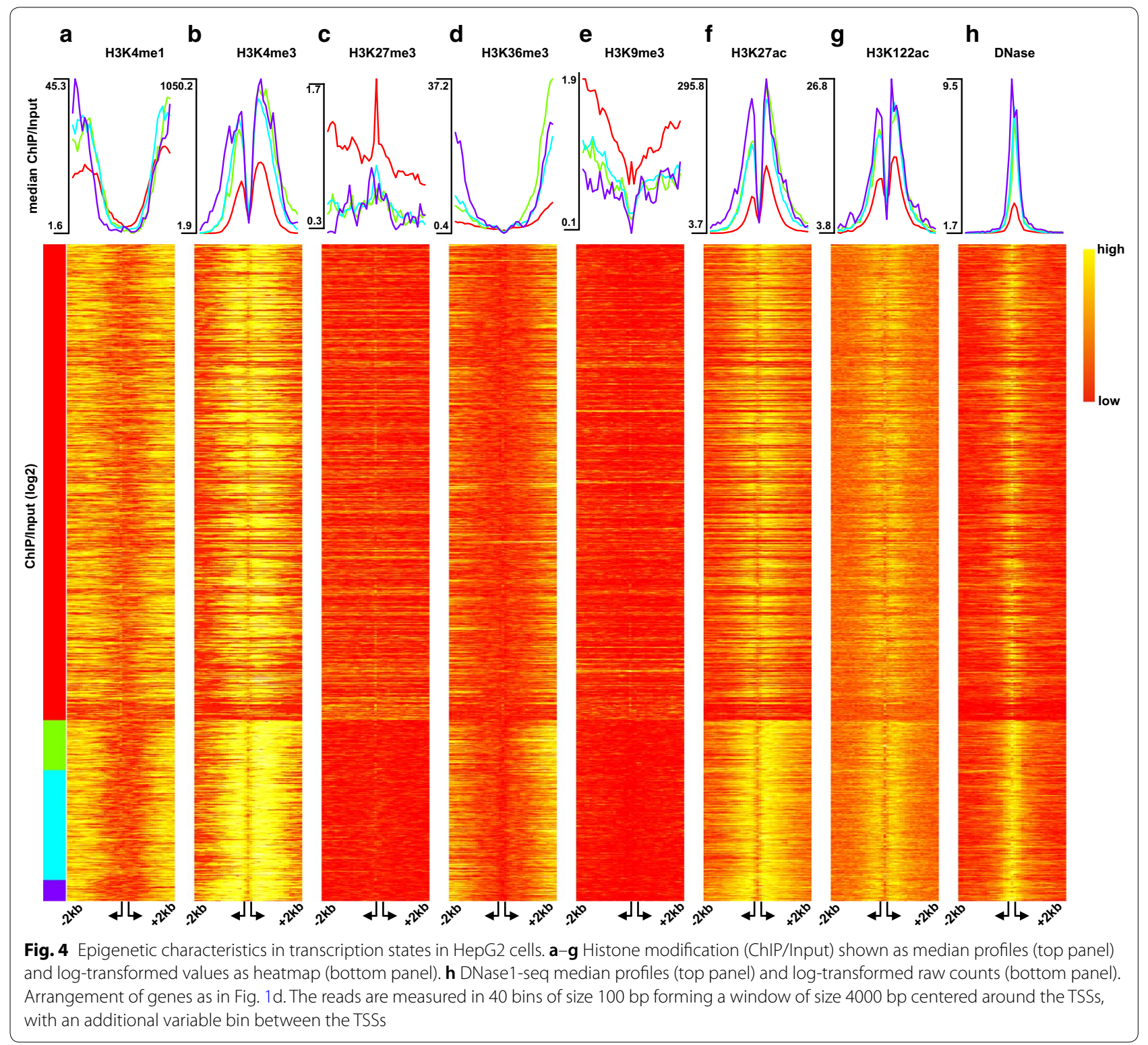

the observation that there is similar amount of single-cell transcription for both genes.

Due to recent reports about small promoter-associated RNAs [24, 25], we obtained small RNA data [19] for HepG2 and K562 samples (see "Methods") and grouped them according to the defined transcription states. Although we observed residual small RNA expression in the vicinity of the bidirectional TSSs, we found no consistent patterns associated with the transcription states (Additional file 1: Fig. S3C).

We also examined the average methylation profiles obtained in the four transcription states (Additional file 1: Materials and Methods) due to the previously reported associations with gene expression $[26,27]$. The results were consistent with other studies where higher level of DNA methylation coincided mostly with silent genes $(B L E)$. Consistent with the enrichment of HMs, genes in the $B N D$ state showed the least amount of DNA methylation (Additional file 1: Fig. S3D).

\section{The BND state coincides with strongest regulatory activity}

It was shown that certain TFs preferentially bind to bidirectional promoters $[13,14]$. As we observed that the DNA accessibility profiles differed among the transcription states (Fig. 4h), we were encouraged to investigate binding of transcription factors. We obtained ChIP-seq data for several transcription factors [19] (44 for HepG2 and 50 for K562). One hypothesis was that there may 
exist TFs that bind in the proximal region of a $\mathrm{BP}$ and influence gene expression as was observed in our transcription states.

To test this, we defined a novel enrichment score tailored to BPs (Additional file 1: Fig. S5A), which preserves the spatial distribution of the ChIP-seq signal along a BP. We applied the enrichment analysis for both cell lines (HepG2 in Fig. 5a and K562 in Additional file 1: Fig. S5B). As expected, states with higher expression showed more TF binding in general. However, we could not pinpoint distinct TF subsets that associate with only one of the states. Instead, the states $B S D, B W D$ and $B N D$ showed enrichment for many of the TFs that we analyzed. We wondered whether the number of TFs that are regulating a BP differed in those states. Figures 5b,c represent the number of positively enriched TFs per BP for each state in both cell lines. The $B N D$ state showed the highest percentage of positively enriched TFs $(t$ test, $p \leq 0.05)$ suggesting that more TFs are required to regulate gene expression in this state.

Next, we tested whether specific genomic regions, such as enhancers, are associated with these four transcription states. For this, we inspected the genome-wide segmentation of HepG2 and K562 cells using an 18-state ChromHMM model [28] (Additional file 1: Fig. S6, Materials and Methods). For simplification we collapsed all TSS-related, enhancer-related, and repression-related ChromHMM states into TSS, Enhancer, and Repressed, respectively. We assigned all the remaining chromatin states to Others (data not shown). The results provided in Figs. 5d,e suggest that the enhancer-related regions are the most frequent among the $B S D$ and $B N D$ states, reflecting their stronger expression profiles. In the case of HepG2 (Fig. 5d), this quantity is even higher than the number of TSS regions. Concurrent with Fig. 4 most of the repressed regions belong to the $B L E$ state, where genes were lowly expressed.

\section{Discussion and conclusions}

In this work we compared single-cell expression of genes at BPs. Currently, we only have access to single-cell protocols for RNA-seq, and other techniques for quantification of transcription start sites cannot be used [4, 6, 29]. Thus, other effects on the mRNA steady-state level, e.g.,
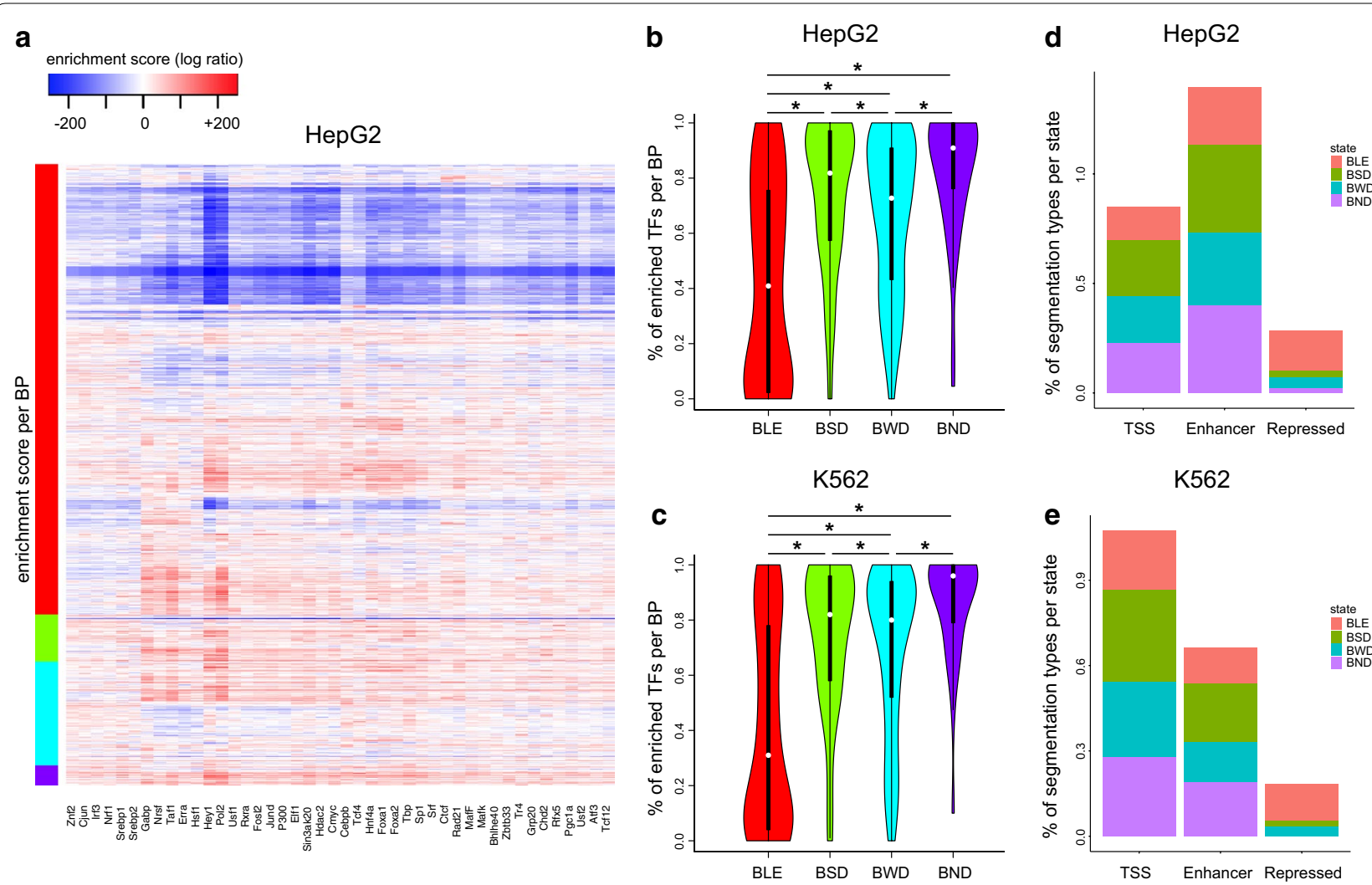

Fig. 5 Transcriptional regulatory features in the transcription states. a Heatmap of TF enrichment scores (log ratio against background) for each BP (row) in HepG2 cells. BPs are sorted as in Fig. 1d. b, c Distributions of percentages of TFs per BP (enrichment score in $\mathbf{a}>0$ ) in each state for HepG2 (top panel) and K562 (bottom panel). d, e ChromHMM annotations, summarized into the types: TSS, Enhancer, and Repressed, are shown as percentages in a bar plot per state (see "Methods") 
post-transcriptional regulation, may influence the gene clustering produced. Here, we have used two high-quality single-cell datasets for ENCODE cell lines allowing us to benefit from a plethora of epigenomic datasets, which are available or have been produced in this work. We found that $88 \%$ of the BPs have the same transcription state in scRNA-seq data despite the difference in origin of HepG2 and K562 cells, which suggests that the majority of these configurations may be stable for many cell types.

In previous work that has analyzed BP regulation, analyses were often limited to a certain configuration at the BP, e.g., a non-coding gene upstream of a coding gene, therefore care has to be taken when comparing to previous studies. Here, we have limited our results to annotated protein- or non-coding genes that originate from a bidirectional promoter. We found that the BPs that show similar expression for both genes are mostly restricted to a configuration with two protein-coding genes. It was shown previously that core promoter strength differs for genes with bidirectional expression and unidirectional promoters [5]. However, we observed that the number of TF regulators that bind to BPs with high bidirectional expression was largest compared to all other expression states we investigated. For this analysis, we used several ChIP-seq datasets for TFs and developed a BP-specific enrichment analysis approach that measures spatial differences in read coverage along the BP regions compared to the median background, unified in a single quantity for each BP and TF. This is different to other studies that have compared TF-ChIP-seq data at BPs, e.g. [14], where the background often were unidirectional promoters rather than all BPs. Thus, to find enrichment in the observed states we properly adjust for the fact that there are two genes, which are regulated by TF binding.

We observed that the $B N D$ state shows the largest (although not strong) single-cell correlation values and that there is a trend with correlation at BP genes being inversely proportional to TSS distance (Fig. 2a, b). A similar observation was recently made for BPs in the rice genome with correlation measured over several bulk RNA-seq datasets [30]. Small distance between the two TSSs may ease the coupled regulation of transcription from both, for example, through a shared or co-regulated Mediator complex [31].

We also found that the transcripts span, the genomic region covered by all transcripts that start in the vicinity of the TSS, was imbalanced for the BSD and BWD states, with the shorter span linked to the highly expressed gene at the BPs. One possibility is that shorter regions of elongation lead to faster transition cycles for Pol2, assuming similar elongation rate of both genes at a BP. This could be a mechanism by the cell to create imbalanced expression output from a shared regulatory region of two BP genes. We also showed that these two states posses the highest percentage of stable and unstable genes inferred by our outlier detection approach. We found out that in these two states only lowly expressed genes were inferred as unstable. As 3'UTR length is found to be associated with regulation of mRNA stability [32], we investigated the $3^{\prime}$ UTR length between the lowly and highly expressed genes in the stable and unstable categories (Additional file 1: Fig. S4C). However, the results showed no apparent significant trend. This probably means that different sets of post-transcriptional regulators are involved in individual bidirectional gene regulation.

Anecdotally, we investigated bulk GRO-cap data for K562 cells [4] and found that the amount of capped nascent transcripts is more similar for both genes at a BP in our states (Additional file 1: Fig. S2E), compared to the amount of fully processed RNAs expressed (CAGE and RNA-seq). Even though the nascent RNA amount is similar, we get significantly different steady-state transcript expression, which could be explained by the difference in length of the genomic region to be elongated, here referred to as transcripts span. Once single-cell measurements of nascent transcription are available, one could investigate the difference in elongation and transcriptional initiation in these BPs.

Taken together, we observed three different genomic and epigenomic architectures underlying single-cell transcription states in BPs. We propose a model depicted in Fig. 6 to describe these architectures. This model supports distinct characteristics of the $B L E$ state, where the bidirectional genes were lowly expressed. They mostly exhibited large TSS distance and more prevalence of repression associated HMs, fewer regions of accessible DNA, and less TF binding. The BSD and BWD states, on the other hand, had reduced TSS distance in comparison with $B L E$ and more abundance of activation associated HMs as well as higher rate of TF binding. Interestingly, the transcripts span associated to the $\mathrm{H}$ gene of BPs in these states was observed to be shorter than the $L$ one. Lastly, $B N D$ showed strongest single-cell co-expression and smallest TSS distance among the states. Furthermore, we observed the widest accessible regions of DNA, the largest number of binding TFs and highest amount of activation related HMs.

Although the transcription state definition was based on the single-cell data, several bulk datasets showed consistent and matching patterns for those states. Our results suggest that novel statistical methods can be developed to deconvolute masked subpopulations of cells measured with different bulk epigenomic assays with the help of single-cell RNA-seq data. Further advances in single-cell sequencing technologies [33] are necessary such that we can measure not only RNA expression, but 


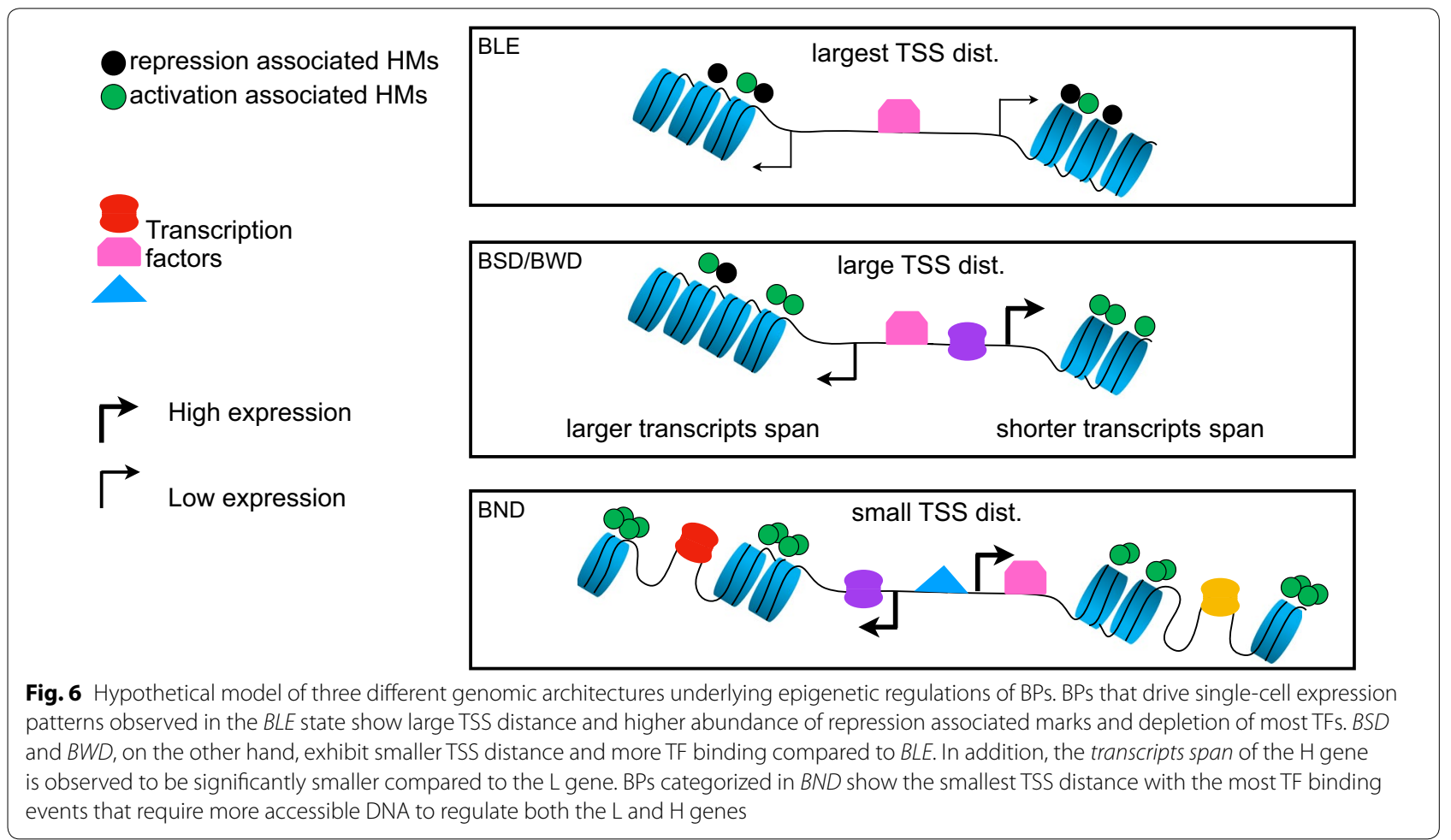

also TF binding and histone modifications in single cells to understand the hidden complexity, in particular, in BP regulation.

\section{Methods}

\section{Datasets and pre-processing}

\section{Single-cell RNA-seq}

Single HepG2 cells were manually picked to prepare poly-A enriched cDNA libraries using Smart-seq 2 as described by [34] with modifications. Briefly, 65 singlecell samples were supplemented with $0.5 \mu \mathrm{l}$ of a 1:40,000 dilution of the Ambion ERCC RNA Spike-In Mix 1 (Thermo Sientific, \#4456740). After cell lysis polyadenylated mRNA was reverse transcribed using a biotinylated template switch oligo (5'-Biotin-AAGCAGTGG TATCAACGCAGAGTACATrGrG+G-3') with two riboguanosines $(\mathrm{rG})$ and one LNA-modified guanosine $(+\mathrm{G})$ at the $3^{\prime}$ end. Preamplified cDNA (18 PCR cycles) was purified with Agencourt Ampure XP Beads (Beckman Coulter, \#A 63881) in a 1:1 ratio. cDNA quality of 8 random samples was assessed on the Agilent 2100 Bioanalyzer (Agilent Technologies, \#G2938C) using the Agilent high-sensitivity DNA kit (Agilent Technologies, \# 50674626). Sequencing libraries were prepared using the Nextera XT DNA Sample Preparation Kit (Illumina, \#FC131 - 1024) with approximately $480 \mathrm{pg}$ of cDNA in a $4 \mu \mathrm{l}$ tagmentation reaction followed by a dual indexing PCR with 9 cycles. Individual single-cell libraries were pooled and purified with $0.8 \times$ Agencourt Ampure XP Beads. The library pool was sequenced on a HiSeq 2500 (Illumina) using the TruSeq SBS Kit v3-HS (Illumina, \#FC401- 3001) in a single read run with $90 \mathrm{bp}$ read length.

\section{Single-cell transcript expression}

The TPM values for transcript isoforms of each Ensembl gene (GRCh37) were computed using RSEM [35]. To attribute the transcription expression to each bidirectional gene, we summed the isoform TPM values of transcripts that had their annotated TSS within a $2 \mathrm{~kb}$ window downstream of the most $5^{\prime}$ TSS of that gene.

\section{HepG2 and $K 562$ datasets}

Epigenomic data for the HepG2 cell lines have been produced by the DEEP consortium and are deposited at the European Genome-Phenome Archive under the accession number EGAS00001001656. The rest of the data, K562 (HM-ChIP-Seq, TF-ChIP-seq, CAGE), and HepG2 (TF-ChIP-seq, CAGE) were obtained from the ENCODE portal.

\section{Bidirectional promoter (BP) gene set}

The BP dataset contained 1242 divergent promoters with two core promoter elements, obtained from annotated ENSEMBL genes (GRCh37.75), such that the distance 
between TSSs of each BP does not exceed $500 \mathrm{bp}$. This set excludes loci overlapped by any other annotated gene region ( $\pm 2 \mathrm{~kb}$ from the TSS).

\section{Clustering BPs into four states}

Hierarchical clustering (TPM values) using the complete linkage method with Euclidean distance as distance metric was applied on the swapped BP matrix using $\mathrm{R}$.

\section{Constructing the single-cell TPM matrix for BPs}

For a particular BP, $\mathrm{BP}_{i}=\left(g_{\text {crick }, i}, g_{\text {watson }, i}\right)$, we compute the sum of TPM values across single cells as following:

$$
\operatorname{Sum}\left(g_{j, i}\right)=\sum_{c=1}^{N} \operatorname{TPM}\left(g_{j, i}^{c}\right),
$$

where $N$ denotes the number of single cells, and $\operatorname{TPM}\left(g_{j, i}^{c}\right)$ returns the TPM value for gene $j \in$ \{crick, watson $\}$ of $\mathrm{BP}_{i}$ in cell c. The orientation of genes at a BP is not specific to the DNA strand, but the lower expressed gene of a BP is always swapped to the left and higher expressed gene to the right. In this way, without loss of generality, all analyses correctly adjust for differences of expression. Precisely, we define $g_{H, i}$ denoting the gene of $\mathrm{BP}_{i}$ having higher expression as follows:

$$
g_{H, i}= \begin{cases}g_{\text {watson }, i}, & \text { if } \operatorname{Sum}\left(g_{\text {watson }, i}\right) \geq \operatorname{Sum}\left(g_{\text {crick }, i}\right) \\ g_{\text {crick }, i}, & \text { else. }\end{cases}
$$

Similarly, we define $g_{L, i}$ denoting the gene of $\mathrm{BP}_{i}$ having lower

sion: $g_{L, i}= \begin{cases}g_{\text {watson }, i}, & \text { if } \operatorname{Sum}\left(g_{\text {watson }, i}\right)<\operatorname{Sum}\left(g_{\text {crick }, i}\right) \\ g_{\text {crick }, i}, & \text { else. }\end{cases}$

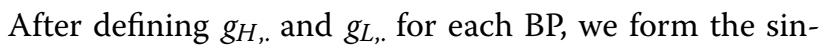
gle-cell matrix for BPs, scBP, as follows:

$$
\mathrm{scBP}=\left[\begin{array}{ccccccc}
g_{L, 1}^{1} & g_{L, 1}^{2} & g_{L, 1}^{N} & g_{H, 1}^{1} & g_{H, 1}^{2} & \ldots & g_{H, 1}^{N} \\
g_{L, 2}^{1} & g_{L, 2}^{2} & g_{L, 2}^{N} & g_{H, 2}^{1} & g_{H, 2}^{2} & \ldots & g_{H, 2}^{N} \\
\vdots & \vdots & \vdots & \ddots & \vdots & \ddots & \vdots \\
g_{L, M}^{1} & g_{L, M}^{2} & g_{L, M}^{N} & g_{H, M}^{1} & g_{H, M}^{2} & \ldots & g_{H, M}^{N}
\end{array}\right]
$$

\section{Imputation of dropouts}

To address the bias caused by dropouts, we performed the most recent and accurate dropout imputation tool called scImpute [36], which aims to improve the singlecell data quality by removing the effects of dropouts without introducing new biases to the data. scImpute has two parameters. $K$ denotes the number of existing cell types in the data, which we set to 1 , as we work on the cell lines. The second parameter $t$ controls the dropout probabilities. The authors show that their results are robust to different parameter values; therefore, we carried on with the default of 0.5 for this parameter. The comparison between raw and imputed read counts performed on the bidirectional genes is shown in Additional file 1: Fig. S1A for both HepG2 and K562. The Pearson correlation between imputed and raw data in both cell lines is $\sim 1$.

\section{Quality of scRNA-seq}

Imputed expression of bidirectional genes averaged over single cells was compared with their corresponding bulk RNA-seq expression. For both, HepG2 and K562, the single-cell expression agrees well with bulk measurements (Spearman correlation coefficient of $\sim 0.8$, Additional file 1: Fig. S1B). Additionally, the imputed TPM values were divided into three intervals, $1<\mathrm{TPM}<10$, $10 \leq \mathrm{TPM} \leq 100, \mathrm{TPM}>100$ to account for the number of genes falling in those intervals per cell (Additional file 1: Fig. S1C, and similarly for the imputed read counts in S1D).

\section{Prediction of RNA stability from histone data}

In order to investigate the potential effects of post-transcriptional regulation on our four transcription states, we exploited the approach proposed by [22], where they predict the RNA expression level using different histone modification ChIP-seq datasets. Similar to their work, we trained an ordinary least squares (OLS) model on all bidirectional genes to predict the average single-cell RNA expression values from the six histone modification datasets we introduced in this manuscript. We designed the input features for the regression such that each histone modification is represented by two bins, one $2 \mathrm{~kb}$ upstream of the TSS and the other $2 \mathrm{~kb}$ downstream (12 features in total). We then fit a linear regression model on our dataset with feature matrix of size $2484 \times 12$ and response vector of size 2484 using the $l m$ function in R, where 2484 is the number of BPs considered. The studentized residuals were computed between the measured average transcript expression values and the predicted values. As suggested by [22], the genes with studentized residuals above 1 or below -1 were annotated as stable or unstable, respectively, and the rest as neutral (Additional file 1: Fig. S4A). We computed the percentage of genes being classified into stable, unstable, or neutral for each state. To assess the enrichment of the stable and unstable mRNAs in each state, we computed the hypergeometric test on the three categories of stable, unstable, and neutral for each state and used the $p \leq 0.05$ as significance cutoff.

\section{Bidirectional gene signature: concordant or discordant}

We define two types of signatures to address the changes in bidirectional gene expression. Intuitively, if the two 
genes are mostly expressed in a consistent manner across the single cells, for example one is always higher than the other, this would be considered as concordant signature. However, if the expression of these two genes flips across cells, we refer to this case as discordant. To analytically differentiate between both signatures for each pair of genes in a BP, we performed the Wilcoxon signed rank test on their imputed single-cell expression (BPs where both genes had zero expression in all cells were removed for the test). If the $p$ value after using Benjamini-Hochberg multiple testing correction is smaller than or equal to 0.05 , the gene pair is considered to be concordant. The number of concordant BPs normalized by the total number of BPs in a given cluster is defined as concordant ratio.

\section{Enrichment of gene products categorized according to transcription states}

We categorized the gene product annotations into two groups, protein-coding (PC) and the rest as non-coding (NC). In the context of BPs, we introduce a new notation, $\mathrm{gp} \in\{\mathrm{NC} \rightarrow \mathrm{NC}, \mathrm{NC} \rightarrow \mathrm{PC}, \mathrm{PC} \rightarrow \mathrm{NC}, \mathrm{PC} \rightarrow \mathrm{PC}\}$, representing the gene products of a pair of genes. We measured the occurrences of each of the above four categories for the gene pairs of our transcription states as shown in Figs. If and Additional file 1: Fig. S2C. To compute the enrichment of such occurrences, we applied a hypergeometric test on their contingency table, $C \in \mathbb{Z}^{4 \times 4}$, where $C_{i, j}$ represents the frequency of the jth gene product category in the ith state. Precisely, let $h(x ; N, n, k)$ be the hypergeometric distribution, where $N$ denotes the population size, $n$ denotes the sample size, $\mathrm{k}$ is the frequency of successes in the population, and $\mathrm{x}$ represents the frequency of successes in the sample. To apply this distribution to each entry $C_{i, j}$ of the contingency matrix $\mathrm{C}$, we used the following setup:

$$
h\left(C_{i, j} ; \sum_{r=1}^{4} \sum_{s=1}^{4} C_{r, s}, \sum_{r=1}^{4} C_{r, j}, \sum_{r=1}^{4} C_{i, r}\right) .
$$

The $p$ value derived from this test was used to quantify the significance of enrichment of a gene product category in a particular state.

\section{Enrichment of TF-ChIP-seq data}

To preserve the spatial distribution of the TF-ChIPseq signal around the promoter, the ChIP-seq reads are counted in bins of size $100 \mathrm{bp}$ forming a window starting at the TSS of each bidirectional gene and extending up to $2000 \mathrm{bp}$ downstream of each of two TSSs (Additional file 1: Fig. S5A). An additional bin with variable size is allocated to count the reads falling within the region between the TSSs of the two bidirectional genes. The 20 bins from the $\mathrm{L}$ gene, the bin for region between both TSSs, and the 20 bins from the $\mathrm{H}$ gene are all combined into one vector of size 41 that represent the binned ChIP-seq signal per BP for a particular TF. To compute the enrichment score of the ith TF at a particular BP, we define:

$$
\operatorname{Enrich}\left(\mathrm{TF}^{i}\right)=\sum_{j=1}^{41} \log _{2}\left(\frac{\mathrm{TF}_{j}^{i}+1}{\mathrm{BG}_{j}^{i}+1}\right),
$$

where $\mathrm{TF}^{i}$ is the signal measured for ith TF (for HepG2, $i \in\{1, \ldots, 44\}$ and for K562, $i \in\{1, \ldots, 50\})$ at the given BP. $\mathrm{TF}_{j}^{i}$ denotes the read counts measured at the $j$ th bin of $\mathrm{TF}^{i}$ signal and $\mathrm{BG}_{j}^{i}$ denotes the median of $\mathrm{TF}^{i}$ signal measured at the $j$ th bin across all BPs.

\section{Definition of transcribed regions}

For each gene, we consider all the annotated transcripts that start within $2 \mathrm{~kb}$ downstream of the most $5^{\prime}$ TSS of the gene. We measured the length of the exonic region encompassed by these transcripts, which we refer to as transcript length. For instance, consider the exonic coordinates of the two transcripts of a gene that start within $2 \mathrm{~kb}$ downstream of the most $5^{\prime}$ TSS, $T_{1}=\left\{\left(E_{\text {start }}^{1}: 0, E_{\text {end }}^{1}: 500\right),\left(E_{\text {start }}^{2}: 600, E_{\text {end }}^{2}: 1000\right)\right\}$, $T_{2}=\left\{\left(E_{\text {start }}^{1}: 0, E_{\text {end }}^{1}: 100\right),\left(E_{\text {start }}^{2}: 400, E_{\text {end }}^{2}: 600\right),\left(E_{\text {start }}^{3}:\right.\right.$ $\left.\left.1500, E_{\text {end }}^{3}: 3000\right)\right\}$, where $E_{\text {start }}^{(.)}$and $E_{\text {end }}^{(.)}$are relative coordinates to the most $5^{\prime}$ TSS. Then the transcripts length would be equal to $2500 \mathrm{bp}$; the length of the region covered by exons $E 1$ and $E 2$ from both transcripts, $1000 \mathrm{bp}$, plus the length of the exon $E 3$ of transcript $T_{2}, 1500 \mathrm{bp}$. Similarly, the exonic and intronic region spanned by those transcripts is referred to as transcripts span. Referring back to the example above, the transcripts span would be equal to (start: 0, end: 3000), where start and end are relative coordinates to the most $5^{\prime}$ TSS. Note that all regions in this interval are considered, regardless of their exonic or intronic annotations. Also note that other transcripts of the gene that would start outside of the $2 \mathrm{~kb}$ region are not considered for the definition of transcripts span or transcript length.

\section{Chromatin state segmentation score}

We acquired the 18-states ChromHMM [28] annotation for both cell lines, for HepG2 produced by DEEP, and for K562 downloaded from Roadmap [37]. For simplicity, we collapsed all TSS-related states to one state called, TSS. Similarly, we defined Enhancer and Repressed states and assigned all the remaining states to Others, yielding four summarized states in general. Later, for each gene $g$ we defined a window, $W_{\mathrm{g}}$, starting at the TSS of the gene and 
extending up to the size of the transcripts span, see above. We then computed the average number of bases having a particular chromatin state, s, overlapping in that window. We called this value ChromScore ${ }_{g}^{s}$, described as follows:

$$
\text { ChromScore }_{g}^{s}=\frac{\sum\left\{|R|: R \subseteq W_{g} \text { and state }(R)=s\right\}}{W_{g}},
$$

where $R$ defines a region in the genome, $|R|$ designates the size of this region, and state $(R)$ denotes the chromatin state assigned by ChromHMM to region $R$. It should be noted that since the ChromHMM state annotation is continuous across the genome, the following equation holds:

$$
\sum_{s \in\{\text { TSS,Enhancer,Repressed,Others }\}} \text { ChromScore }_{g}^{s}=1,
$$

and thus ChromScore is properly normalized to account for a difference in transcripts span per gene. To assign ChromScore to a cluster of genes, $\mathrm{C}$, (defining the four transcription states introduced earlier), we formulated the following:

$$
\text { ChromScore }_{C}^{s}=\sum_{g \in C} \text { ChromScore }_{g}^{s},
$$

Later, as the last step, we convert the ChromScore ${ }_{C}^{s}$ into percentages to make the scorecomparable across different clusters of genes with different gene sizes:

$$
\begin{aligned}
& \text { percent(ChromScore } \left.{ }_{C}^{s}\right) \\
& =\frac{\text { ChromScore }_{C}^{s}}{\sum_{s \in\{\text { TSS,Enhancer,Repressed,Others }\}} \text { ChromScore }_{C}^{s}} .
\end{aligned}
$$

\section{Additional file}

Additional file 1. Supplementary methods and results.

\section{Authors' contributions}

FBA was involved in all computational analysis supported by KN and JF; KK and SF generated the single-cell RNA-seq data; NG, GG and KN generated and processed Dnase 1-seq and DNA methylation data; KK generated ChIP-seq data; $\mathrm{MB}, \mathrm{AS}$ and $\mathrm{PR}$ generated and processed bulk RNA-seq data; BF, GZ, KN, $P E, B H, B B$, JE performed mapping and management of sequencing data; $P E$ generated ChromHMM segmentation; MHS designed and coordinated the study supported by FBA, NG, JW, GG, TL, TM. MHS and FBA wrote the manuscript with contributions from other authors.

\section{Author details}

${ }^{1}$ Excellence Cluster for Multimodal Computing and Interaction, Saarland Informatics Campus, Saarland University, Campus E1 7, Saarbrücken 66123, Germany. ${ }^{2}$ Department of Computational Biology and Applied Algorithmics, Max Planck Institute for Informatics, Saarland Informatics, Campus E
4, Saarbrücken 66123, Germany. ${ }^{3}$ Graduate School of Computer Science, Saarland University, Campus E1 3, Saarbrücken 66123, Germany. ${ }^{4}$ Department of Genetics, University of Saarland, Campus A2 4, Saarbrücken 66123, Germany. ${ }^{5}$ Max Planck Institute of Immunobiology and Epigenetics, Stübeweg 51, Freiburg 79108, Germany. ${ }^{6}$ Institute of Clinical Molecular Biology, Christian-Albrechts-University, Rosalind-Franklin-Str. 12, Kiel 24105, Germany. ${ }^{7}$ Applied Bioinformatics, Deutsches Krebsforschungszentrum, Berliner-Str. 41, Heidelberg 69120, Germany. ${ }^{8}$ Data Management and Genomics IT, Deutsches Krebsforschungszentrum, Im Neuenheimer Feld 280, Heidelberg 69120, Germany. ${ }^{9}$ Institute for Cardiovascular Regeneration, Goethe University, Theodor-Stern-Kai 7, Frankfurt am Main 60590, Germany. ${ }^{10}$ German Center for Cardiovascular Research, Partner site Rhein-Main, Frankfurt am Main 60590, Germany.

\section{Acknowledgements}

We thank Alex A Pollen and the SRA archive for help with obtaining the singlecell data for K562. We are thankful to all labs that contributed to the ENCODE data used.

\section{Competing interests}

The authors declare that they have no competing interests.

\section{Availability of data and materials}

All sequencing DEEP data used for this study have been deposited at the European Genome-Phenome Archive under the accession number EGAS00001001656. The source code for the conducted analyses and code for making the figures can be found in the GitHub repository via https://githu b.com/SchulzLab/scBP.

\section{Consent for publication}

Not applicable.

\section{Ethics approval and consent to participate} Not applicable.

\section{Funding}

German Epigenome Programme (DEEP) of the Federal Ministry of Education and Research in Germany (BMBF) 01KU1216F to JW and 01KU1216A to PE.

\section{Publisher's Note}

Springer Nature remains neutral with regard to jurisdictional claims in published maps and institutional affiliations.

Received: 2 May 2018 Accepted: 26 October 2018

Published online: 10 November 2018

\section{References}

1. Core LJ, Waterfall JJ, Lis JT. Nascent RNA sequencing reveals widespread pausing and divergent initiation at human promoters. Science. 2008;322(5909):1845-8. https://doi.org/10.1126/science.1162228.

2. Preker P, Nielsen J, Kammler S, Lykke-Andersen S, Christensen MS, Mapendano CK, Schierup MH, Jensen TH. RNA Exosome depletion reveals transcription upstream of active human promoters. Science. 2008;322(5909):1851-4. https://doi.org/10.1126/science.1164096.

3. Seila AC, Calabrese JM, Levine SS, Yeo GW, Rahl PB, Flynn RA, Young RA, Sharp PA. Divergent transcription from active promoters. Science. 2008;322(5909):1849-51. https://doi.org/10.1126/science.1162253.

4. Core LJ, Martins AL, Danko CG, Waters CT, Siepel A, Lis JT. Analysis of nascent RNA identifies a unified architecture of initiation regions at mammalian promoters and enhancers. Nat Genet. 2014;46(12):1311-20.

5. Duttke SHC, Lacadie SA, Ibrahim MM, Glass CK, Corcoran DL, Benner C, Heinz S, Kadonaga JT, Ohler U. Human promoters are intrinsically directional. Mol Cell. 2015;57(4):674-84.

6. Scruggs BS, Gilchrist DA, Nechaev S, Muse GW, Burkholder A, Fargo DC, Adelman K. Bidirectional transcription arises from two distinct hubs of transcription factor binding and active chromatin. Mol Cell. 2015;58(6):1101-12. 
7. Andersson R, Chen Y, Core L, Lis JT, Sandelin A, Jensen TH. Human gene promoters are intrinsically bidirectional. Mol Cell. 2015;60(3):346-7. https ://doi.org/10.1016/j.molcel.2015.10.015.

8. Duttke SHC, Lacadie SA, Ibrahim MM, Glass CK, Corcoran DL, Benner C, Heinz S, Kadonaga JT, Ohler U. Perspectives on unidirectional versus divergent transcription. Mol Cell. 2015;60(3):348-9.

9. Bagchi DN, lyer VR. The determinants of directionality in transcriptional initiation. Trends Genet. 2016;32(6):333. https://doi.org/10.1016/j. tig.2016.03.005.

10. Lacadie SA, Ibrahim MM, Gokhale SA, Ohler U. Divergent transcription and epigenetic directionality of human promoters. FEBS J. 2016. https:// doi.org/10.1111/febs.13747.

11. Lin JM, Collins PJ, Trinklein ND, Fu Y, Xi H, Myers RM, Weng Z. Transcription factor binding and modified histones in human bidirectional promoters. Genome Res. 2007;17(6):818-27.

12. Collins PJ, Kobayashi Y, Nguyen L, Trinklein ND, Myers RM. The ets-related transcription factor GABP directs bidirectional transcription. PLoS Genet. 2007;3(11):208

13. Trinklein ND, Aldred SF, Hartman SJ, Schroeder DI, Otillar RP, Myers RM. An abundance of bidirectional promoters in the human genome. Genome Res. 2004;14(1):62-6. https://doi.org/10.1101/gr.1982804.

14. Bornelöv S, Komorowski J, Wadelius C. Different distribution of histone modifications in genes with unidirectional and bidirectional transcription and a role of CTCF and cohesin in directing transcription. BMC Genom. 2015;16(1):300

15. Marinov GK, Williams BA, McCue K, Schroth GP, Gertz J, Myers RM, Wold BJ. From single-cell to cell-pool transcriptomes: stochasticity in gene expression and RNA splicing. Genome Res. 2013;24(3):496-510.

16. Wu AR, Neff NF, Kalisky T, Dalerba P, Treutlein B, Rothenberg ME, Mburu FM, Mantalas GL, Sim S, Clarke MF, Quake SR. Quantitative assessment of single-cell RNA-sequencing methods. Nat Meth. 2014;11(1):41-6.

17. Pollen AA, Nowakowski TJ, Shuga J, Wang X, Leyrat AA, Lui JH, Li N, Szpankowski L, Fowler B, Chen P, Ramalingam N, Sun G, Thu M, Norris M, Lebofsky R, Toppani D, Kemp DW, Wong M, Clerkson B, Jones BN, Wu S, Knutsson L, Alvarado B, Wang J, Weaver LS, May AP, Jones RC, Unger $M A$, Kriegstein AR, West JAA. Low-coverage single-cell mRNA sequencing reveals cellular heterogeneity and activated signaling pathways in developing cerebral cortex. Nat Biotechnol. 2014;32(10):1053-8.

18. Stunnenberg $\mathrm{HG}$, Hirst $\mathrm{M}$, Consortium IHE, et al. The international human epigenome consortium: a blueprint for scientific collaboration and discovery. Cell. 2016;167(5):1145-9.

19. ENCODEConsortium. An integrated encyclopedia of DNA elements in the human genome. Nature. 2012;489(7414):57-74.

20. Bannister AJ, Kouzarides T. Regulation of chromatin by histone modifications. Cell Res. 2011;21(3):381-95. https://doi.org/10.1038/cr.2011.22.

21. Sein H, Värv S, Kristjuhan A. Distribution and maintenance of histone h3 lysine 36 trimethylation in transcribed locus. PLoS ONE. 2015;10(3):1-10 https://doi.org/10.1371/journal.pone.0120200.

22. Wang C, Tian R, Zhao Q, Xu H, Meyer CA, Li C, Zhang Y, Liu XS. Computational inference of mRNA stability from histone modification and transcriptome profiles. Nucleic Acids Res. 2012;40(14):6414-23. https:// doi.org/10.1093/nar/gks304.

23. Clark MB, Johnston RL, Inostroza-Ponta M, Fox AH, Fortini E, Moscato P, Dinger ME, Mattick JS. Genome-wide analysis of long noncoding RNA stability. Genome Res. 2012;22(5):885-98. https://doi.org/10.1101/ gr.131037.111.

24. Zamudio JR, Kelly TJ, Sharp PA. Argonaute-bound small rnas from promoter-proximal rna polymerase ii. Cell. 2014;156(5):920-34. https:// doi.org/10.1016/j.cell.2014.01.041.

25. Wang C, Ge Q, Chen Z, Hu J, Li F, Ye Z. Promoter-associated endogenous and exogenous small RNAs suppress human bladder cancer cell metastasis by activating p21 CIP1/WAF1 expression. Tumor Biology. 2016;37(5):6589-98. https://doi.org/10.1007/s13277-015-4571-z.
26. Siegfried Z, Simon I. Dna methylation and gene expression. Wiley Interdiscip Rev Syst Biol Med. 2010;2(3):362-71. https://doi.org/10.1002/ wsbm.64.

27. Schübeler D. Function and information content of DNA methylation. Nature. 2015;517(7534):321-6.

28. Ernst, J, Kellis, M. ChromHMM automating chromatin-state discovery and characterization. Nat Methods. 2012. https://doi.org/10.1093/nar/gkv14 95

29. Shiraki T, Kondo S, Katayama S, Waki K, Kasukawa T, Kawaji H, Kodzius R, Watahiki A, Nakamura M, Arakawa T, Fukuda S, Sasaki D, Podhajska A, Harbers M, Kawai J, Carninci P, Hayashizaki Y. Cap analysis gene expression for high-throughput analysis of transcriptional starting point and identification of promoter usage. Proc Natl Acad Sci USA. 2003;100(26):15776-81. https://doi.org/10.1073/pnas.2136655100.

30. Fang $Y$, Wang $L$, Wang $X$, You Q, Pan $X$, Xiao J, Wang X-E, Wu Y, Su Z, Zhang W. Histone modifications facilitate the coexpression of bidirectional promoters in rice. BMC Genom. 2016;17(1):768. https://doi.org/10.1186/ s12864-016-3125-0.

31. Allen BL, Taatjes DJ. The Mediator complex: a central integrator of transcription. Nat Rev Mol Cell Biol. 2015;16(3):155-66. https://doi. org/10.1038/nrm3951.

32. Mayr C. Regulation by $3^{\prime}$-untranslated regions. Annu Rev Genet. 2017;51(1):171-94. https://doi.org/10.1146/annurev-genet-120116-02470 4.

33. Schwartzman O, Tanay A. Single-cell epigenomics: techniques and emerging applications. Nat Rev Genet. 2015;16(12):716-26.

34. Picelli S, Faridani OR, Björklund AK, Winberg G, Sagasser S, Sandberg R. Full-length RNA-seq from single cells using Smart-seq2. Nat Protoc. 2014;9:171-81. https://doi.org/10.1038/nprot.2014.006.

35. Li B, Dewey CN. RSEM: accurate transcript quantification from RNA-Seq data with or without a reference genome. BMC Bioinform. 2011;12(1):323. https://doi.org/10.1186/1471-2105-12-323.

36. LiWV, Li JJ. An accurate and robust imputation method sclmpute for single-cell RNA-seq data. Nat Commun. 2018;9(1):997. https://doi. org/10.1038/s41467-018-03405-7.

37. Consortium RE, Kundaje A, Meuleman W, Ernst J, Bilenky M, Yen A, Moussavi AH, Kheradpour P, Zhang Z, Wang J, Ziller MJ, Amin V, Whitaker JW, Schultz MD, Ward LD, Sarkar A, Quon G, Sandstrom RS, Eaton ML, Wu Y, Pfenning AR, Wang X, Claussnitzer M, Liu Y, Coarfa C, Harris RA, Shoresh N, Epstein CB, Gjoneska E, Leung D, Xie W, Hawkins RD, Lister R, Hong C, Gascard P, Mungall AJ, Moore RA, Chuah E, Tam A, Canfield TK, Hansen RS, Kaul R, Sabo PJ, Bansal MS, Carles A, Dixon JR, Farh K, Feizi S, Karlic R, Kim A, Kulkarni A, Li D, Lowdon RF, Elliott G, Mercer TR, Neph SJ, Onuchic V, Polak P, Rajagopal N, Ray P, Sallari RC, Siebenthall KT, Sinnott-Armstrong NA, Stevens M, Thurman RE, Wu J, Zhang B, Zhou X, Beaudet AE, Boyer LA Jager PLD, Farnham PJ, Fisher SJ, Haussler D, Jones SJM, Li W, Marra MA, McManus MT, Sunyaev SR, Thomson JA, TIsty TD, Tsai L, Wang W, Waterland RA, Zhang MQ, Chadwick LH, Bernstein BE, Costello JF, Ecker JR, Hirst M, Meissner A, Milosavljevic A, Ren B, Stamatoyannopoulos JA, Wang T, Kellis M. Integrative analysis of 111 reference human epigenomes open. Nature. 2015;518:317-30. https://doi.org/10.1038/nature14248.

Ready to submit your research? Choose BMC and benefit from:

- fast, convenient online submission

- thorough peer review by experienced researchers in your field

- rapid publication on acceptance

- support for research data, including large and complex data types

- gold Open Access which fosters wider collaboration and increased citations

- maximum visibility for your research: over $100 \mathrm{M}$ website views per year

At BMC, research is always in progress.

Learn more biomedcentral.com/submissions 